\title{
Effects of the ninein-like protein centrosomal protein on breast cancer cell invasion and migration
}

\author{
QI LIU $^{1 *}$, XINZHAO WANG ${ }^{1 *}$, MINLIN LV ${ }^{2}$, DIANBIN MU ${ }^{1}$, LEILEI WANG $^{1}$, \\ WENSU ZUO ${ }^{1}$ and ZHIYONG YU ${ }^{1}$ \\ ${ }^{1}$ Department of II, Shandong Breast Center of Prevention and Treatment, Shandong Cancer Hospital, Jinan, Shandong 250117; \\ ${ }^{2}$ Clinical Laboratory, Haiyang People's Hospital, Yantai, Shandong 265100, P.R. China
}

Received June 8, 2014; Accepted February 17, 2015

DOI: $10.3892 / \mathrm{mmr} .2015 .3650$

\begin{abstract}
To investigate the effects of the centrosomal protein, ninein-like protein (Nlp), on the proliferation, invasion and metastasis of MCF-7 breast cancer cells, the present study established green fluorescent protein (GFP)-containing MCF7 plasmids with steady and overexpression of Nlp (MCG7-GFP-N1p) and blank plasmids (MCF7-GFP) using lentiviral transfection technology in MCF7 the breast cancer cell line. The expression of Nlp was determined by reverse transcription-quantitative polymerase chain reaction and western blott analysis. Differences in levels of proliferation, invasion and metastasis between the MCF7-GFP-Nlp group and MCF-GFP group were compared using MTT, plate colony formation and Transwell migration assays. The cell growth was more rapid and the colony forming rate was markedly increased in the MCF7-GFP-Nlp group $(\mathrm{P}<0.05)$ compared with the MCF7-GFP group. The number of cells in the MCF-GFP-Nlp and MCF7-GFP groups transferred across membranes were $878 \pm 18.22$ and $398 \pm 8.02$, respectively, in the migration assay. The invasive capacity was significantly increased in the MCF7-GFP-Nlp group $(\mathrm{P}<0.05)$ compared with the MCF7-GFP group. The western blotting results demonstrated high expression levels of $\mathrm{C}-\mathrm{X}-\mathrm{C}$ chemokine receptor type 4 in the MCF7-GFP-Nlp group. The increased expression of Nlp was associated with an increase in MCF7 cell proliferation, invasion and metastasis, which indicated that Nlp promoted breast tumorigenesis and may be used as a potent biological index to predict breast cancer metastasis and develop therapeutic regimes.
\end{abstract}

Correspondence to: Mr. Zhiyong Yu, Department of II, Shandong Breast Center of Prevention and Treatment, Shandong Cancer Hospital, 440 Jiyan Road, Huaiyin, Jinan, Shandong 250117, P.R. China

E-mail: zhiyongyudoc@126.com

*Contributed equally

Key words: breast cancer, centrosomal protein, ninein-like protein, tumor invasion, tumor metastasis

\section{Introduction}

Breast cancer is a malignant tumor, which severely affects female health, is life threatening and the incidence of which has increased gradually over recent years $(1,2)$. With the prevalence of advanced diagnostic instruments and the development of standardized systematic therapy, the rate of early diagnosis in patients with recurrent-metastasis patients has increased and survival rates have improved, with mortality rates declining by $1-2 \%$ per year in China $(1,2)$. The present study aimed to investigate the risk factors of breast cancer, recurrent-metastasis and intervention methods, which are important to decrease the breast cancer mortality rate. The human centrosomal ninein-like protein (Nlp) is a novel member of the $\gamma$-tubulin complex binding proteins (GTBPs) and is essential in the process of mitosis. The primary function of Nlp is to promote microtubule nucleation, which contributes to centrosomal maturation, spindle formation and chromosome segregation $(3,4)$. The centrosome from almost all types of tumor exhibit abnormal structure, morphology and function. Previous studies have demonstrated that centrosome activity is important in cell division and in the transition from G1 phase to $\mathrm{S}$ phase $(5,6)$. Abnormal centrosomes may lead to interruption of the cell cycle, including the polycaryon phenotype, which causes abnormal cell transformation, tumorigenesis and the development of malignancy (7-9). In the present study, the biological action of Nlp on metastatic capacity of breast cancer was investigated using advanced transfection technology.

\section{Materials and methods}

Cell culture. The MCF-7 breast cancer cell line was provided by the Basic Center of Shandong Tumor Hospital (Shandong, China), and the cells were cultured in Dulbecco's modified Eagle's medium (DMEM; Hyclone Corporation, Logan, USA), containing 10\% fetal bovine serum (Hyclone Corporation), $100 \mathrm{U} / \mathrm{ml}$ penicillin and $100 \mathrm{pg} / \mathrm{ml}$ streptomycin (Beyotime Institute of Biotechnology, Haimen, China) in a humidified incubator at $37^{\circ} \mathrm{C}$ under $5 \% \mathrm{CO}_{2}$.

Steady transfection with lentivirus. The MCF-7 cells were seeded into $10 \mathrm{~cm}$ cell culture bottles $\left(6 \times 10^{5}\right.$ cells $\left./ \mathrm{ml}\right)$ containing $10 \%$ serum and medium $24 \mathrm{~h}$ prior to transfection 
and were cultured in a humidified incubator at $37^{\circ} \mathrm{C}$ under $5 \% \mathrm{CO}_{2}$. The cells were not transfected until the cell density was between 70 and $80 \%$ confluent. The culture medium was replaced with medium without serum $2 \mathrm{~h}$ prior to transfection. The prepared DNA solution, containing either an enhanced green fluorescent protein (EGFP)-C1 plasmid $(40 \mu \mathrm{l})$ or a pEGFP-C1-Nlp plasmid (100 $\mu$; B Beijing Dingguo Changsheng Biotechnology Co., Ltd., Beijing, China) was added to the tubes and mixed with Opti-MEM medium to a final volume of $2.5 \mathrm{ml}$ (Gibco Life Technologies, Carlsbad, CA, USA). Following addition of the transfection reagent, Attractene (Qiagen, Hilden, Germany) and agitating lightly, Opti-MEM medium was added for $20 \mathrm{~min}$ at room temperature. The transfection mixture was transferred into medium containing $5 \times 10^{5}$ MCF-7 cells and cultured for $8 \mathrm{~h}$ in a humidified incubator at $37^{\circ} \mathrm{C}$ under $5 \% \mathrm{CO}_{2}$. Following incubation, the medium was replaced with $25 \mathrm{ml}$ fresh $10 \%$ serum containing medium for $48 \mathrm{~h}$ in a humidified incubator at $37^{\circ} \mathrm{C}$ under $5 \% \mathrm{CO}_{2}$. The MCF-7 supernatant was centrifuged at $4,000 \mathrm{x} \mathrm{g}$ for $10 \mathrm{~min}$ at $4^{\circ} \mathrm{C}$ and transferred to a filter cup (Sartorius, Goettingen, Germany). The filter cup was then inserted into a filtrate collection tube (Sartorius). The lentivirus was centrifuged at $1,000 \mathrm{x} \mathrm{g}$ for $2 \mathrm{~min}$ at $4^{\circ} \mathrm{C}$ and stored at $-80^{\circ} \mathrm{C}$,

mRNA expression of Nlp was detected by reverse transcription-quantitative polymerase chain reaction ( $R T-q P C R)$. The total RNA was extracted using TRIzol reagent (Invitrogen Life Technologies, Carlsbad, CA, USA), according to the manufacturer's instructions. The purity and concentration of the RNA samples were determined using an ultraviolet spectrophotometer (DU 800; Beckman Coulter, Brea, CA, USA) and samples with an absorbance value $>1.7$ were assessed by RT-qPCR. A total of $1 \mu \mathrm{g}$ RNA was used to produce cDNA according to the manufacturer's instructions of the TUREscript 1st Strand cDNA Synthesis kit (Aidlab Biotechnologies Co., Ltd., Beijing, China). The cDNA reaction system was as follows: $1 \mu \mathrm{l}$ of Oligo(dt)18 $(0.5 \mu \mathrm{g} / \mu \mathrm{l}), 10 \mu \mathrm{l}$ of $2 \mathrm{X}$ RT Reaction mix, $1 \mu \mathrm{l}$ of TUREscript H-RTase/RI mix and RNase free $\mathrm{H}_{2} \mathrm{O}$ at a final volume of $20 \mu \mathrm{l}$. The RT-qPCR assays were performed according to the manufacturer's instructions of the $2 X$ SYBR Green qPCR kit (Aidlab Biotechnologies Co., Ltd., Beijing, China). PCR was conducted using an Applied Biosystems ABI Prism 7000 Real-Time PCR System (Applied Biosystems, Foster city, CA, USA). The cycling conditions were as follows: $94^{\circ} \mathrm{C}$ for $3 \mathrm{~min}$ to activate the DNA polymerase, followed by 40 cycles of $95^{\circ} \mathrm{C}$ for $40 \mathrm{sec}, 61^{\circ} \mathrm{C}$ for $60 \mathrm{sec}$ and $72^{\circ} \mathrm{C}$ for $40 \mathrm{sec}$, and then extended at $72^{\circ} \mathrm{C}$ for $10 \mathrm{~min}$. The specificity of the amplification products were confirmed by melting curve analysis. The PCR reactions for each gene were repeated three times and independent experiments were performed in triplicate. The primer (Shanghai Jingmei Bioengineering Co., Ltd., Shanghai, China) sequences were as follows: Nlp, forward 5'-ACCTGGGATTCTGAGGACTTTG-3' and reverse 5'-ACTTTGCCGTCTCCGTCTTGAT-3' and GAPDH, forward 5'-CATCAAGAAGGTGGTGAAGC-3' and reverse 5'-GGAAATTGTGAGGGAGATGC-3'. GAPDH was used as an internal loading control.

Western blotting to detect the protein expression of Nlp and CXCR4. The cells were washed with phosphate-buffered saline (PBS) twice and lysed in radioimmunoprecipitation buffer (50 mM Tris, pH 7.4; $0.15 \mathrm{M} \mathrm{NaCl}$; 1\% Triton X-100; 1\% sodium deoxycholate; $0.1 \%$ SDS) (Aidlab Biotechnologies Co., Ltd.) for $30 \mathrm{~min}$ on ice. The samples were then centrifuged at $12,000 \mathrm{x}$ g for $3 \mathrm{~min}$ at $4^{\circ} \mathrm{C}$ and the supernatants were collected and stored at $-80^{\circ} \mathrm{C}$. Protein concentrations were determined using a bicinchoninic acid assay (Aidlab Biotechnologies Co., Ltd.). The total protein (100 $\mu \mathrm{g})$ was resolved using a $10 \%$ SDS-PAGE gel (Beyotime Institute of Biotechnology), electrotransferred onto polyvinylidene fluoride membranes (GE Healthcare Life Sciences, Little Chalfont, UK) and blocked with 5\% non-fat dry milk (Shanghai Bright Dairy Group Co., Ltd., Shanghai, China) for $1 \mathrm{~h}$ in Tris-buffered saline (Boster Biological Technology, Ltd., Wuhan, China). The membranes were incubated in primary Nlp polyclonal antibody (1:1,000; cat. no. ab179678; Abcam, Cambridge, CA, USA), rabbit polyclonal C-X-C chemokine receptor (CXCR)4 (1:2,000; cat. no. ab2074; Abcam) and rabbit polycloanl actin antibody (1:5,000; cat. no. sc-7210; Santa Cruz Biotechnology, Inc., Dallas, TX, USA) overnight at $4^{\circ} \mathrm{C}$. The membranes were washed with PBS, containing $5 \%$ non-fat milk and $0.1 \%$ Tween-20, and were subsequently incubated with horseradish peroxidase-conjugated goat anti-rabbit immunoglobulin G antibody (1:2,000; cat. no. SN134; SunShine Bio, Nanjing, China) for $1 \mathrm{~h}$ at room temperature. Finally, the membranes were incubated with enhanced chemiluminescence reagents (EMD Millipore, Billerica, MA, USA) for $1 \mathrm{~min}$ at room temperature, and developed in a dark room.

MTT assay to detect cell growth curves. The cells were seeded into 96-well plates (1,000 cells/well) and six wells were repeated. The cells were incubated for $24 \mathrm{~h}$ and subsequently treated with $20 \mu \mathrm{l} 5 \mathrm{~g} / \mathrm{l} \mathrm{MTT}$ and incubated at $37^{\circ} \mathrm{C}$ for $4 \mathrm{~h}$. The supernatants were washed and $150 \mu \mathrm{l}$ dimethylsulfoxide (Sigma-Aldrich, St. Louis, MO, USA) was added to each well to terminate the reaction. The optical density (OD) value was detected at a wavelength of $570 \mathrm{~nm}$ on an enzyme-linked immune detector (Model 680; Bio-Rad Laboratories, Inc., Hercules, CA, USA). The OD value was continuously measured for 6 days, and the growth curves were produced. The day was set as abscissa and the absorbance value as the longitudinal coordinates.

Plate colony formation assay. The cells were collected as a single cell suspension and were seeded into 6-well plates, each group had three repeated wells and contained 150-200 cells/well. The single cell suspension was incubated in a humidified incubator at $37^{\circ} \mathrm{C}$ under $5 \% \mathrm{CO}_{2}$ for 12 days, until the cell colonies were visible by eye. The cells were washed twice with PBS and fixed in $100 \%$ methanol (Shenyang Chemical Co., Ltd., Shenyang, China) for $30 \mathrm{~min}$ at room temperature. Following fixation, the cells were stained with $0.5 \%$ crystal violet (Beyotime Institute of Biotechnology) for $5 \mathrm{~min}$, prior to washing and drying. Positive colonies, containing $>50$ cells, were observed under a microscope (IX71; Olympus Corporation, Tokyo, Japan), images were captured and colony numbers were quantified.

Transwell chamber migration assay. For the cell migration assay, $3 \times 10^{5}$ cells in $1 \mathrm{ml}$ medium without fetal calf serum (FCS) were seeded onto a fibronectin coated polycarbonate 


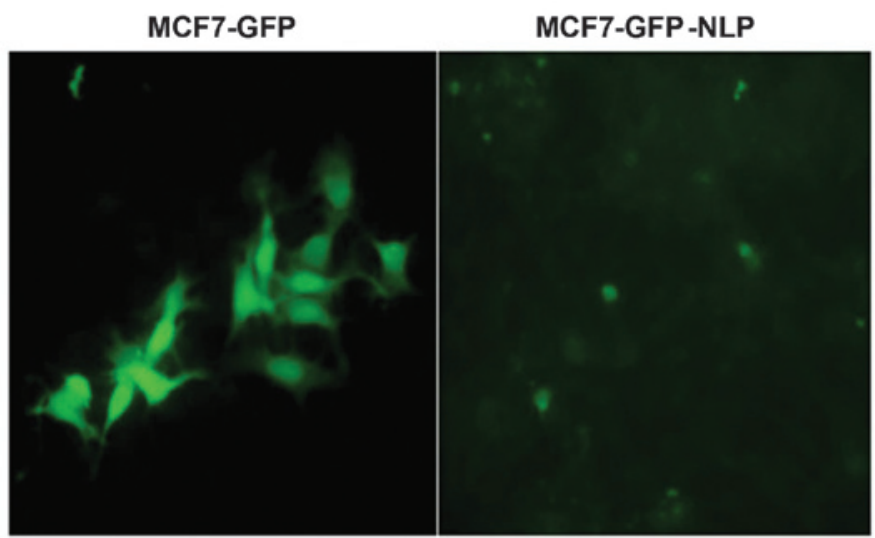

Figure 1. Visualization of the effect of transfection under a fluorescent microscope. When MCF-7 breast cancer cells were transfected with NLP, the transfection efficiency reached $90 \%$, as determined by measuring green fluorescence. (Magnification, x200). GFP, green fluorescent protein; NLP, ninein-like protein.
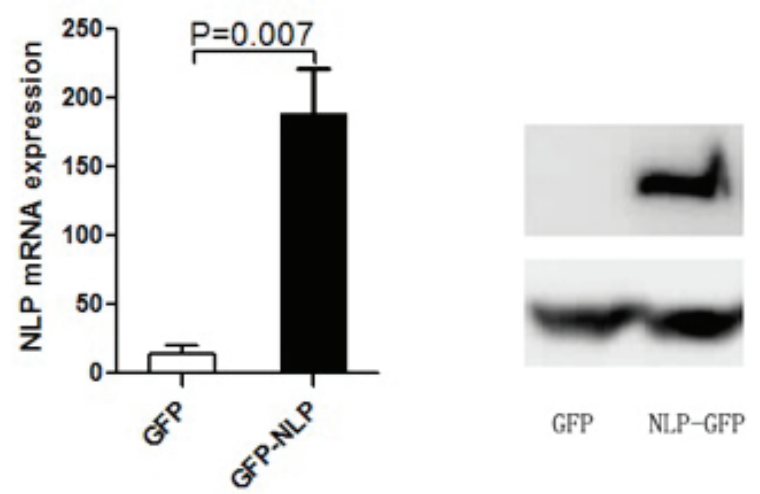

NLP

actin

Figure 2. mRNA and protein expression levels of Nlp in the MCF7-GFP-NLP are significantly increased following transfection. The data are expressed as the mean \pm standard error of the mean. GFP, green fluorescent protein; NLP, ninein-like protein.

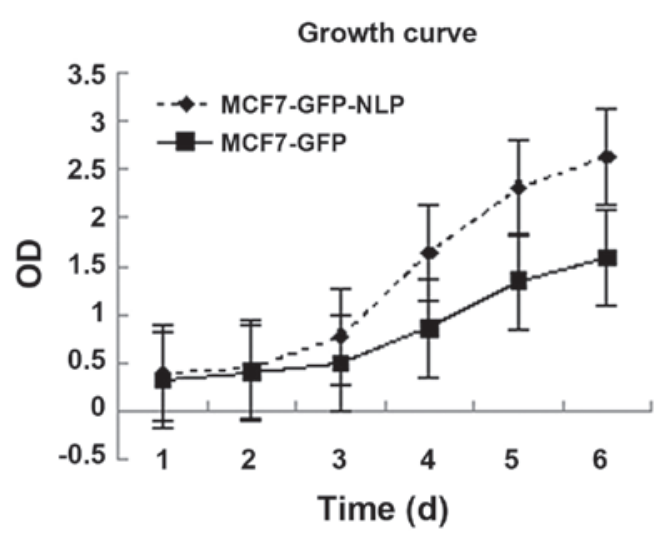

Figure 3. Increased expression levels of Nlp promotes MCF-7 growth $(\mathrm{P}=0.036)$. The data are expressed as the mean \pm standard error of the mean. GFP, green fluorescent protein; Nlp, ninein-like protein; OD, optical density; d, days.

membrane insert in a Transwell apparatus (Corning, Inc., Corning, NY, USA). In the lower chamber, $600 \mu 1$ DMEM, containing $20 \%$ FCS was added as chemoattractant. The cells were incubated for $24 \mathrm{~h}$ at $37^{\circ} \mathrm{C}$ in a $5 \% \mathrm{CO}_{2}$ atmosphere, the insert was washed with PBS and the cells on the top surface of the insert were removed using a cotton swab. The cells

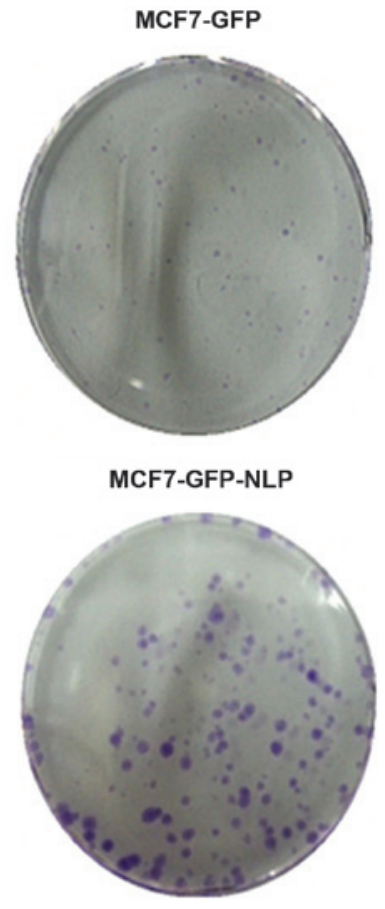

Figure 4. Colony formation in MCF7-GFP cells and MCF7-GFP-NLP cells. GFP, green fluorescent protein; NLP, ninein-like protein. 


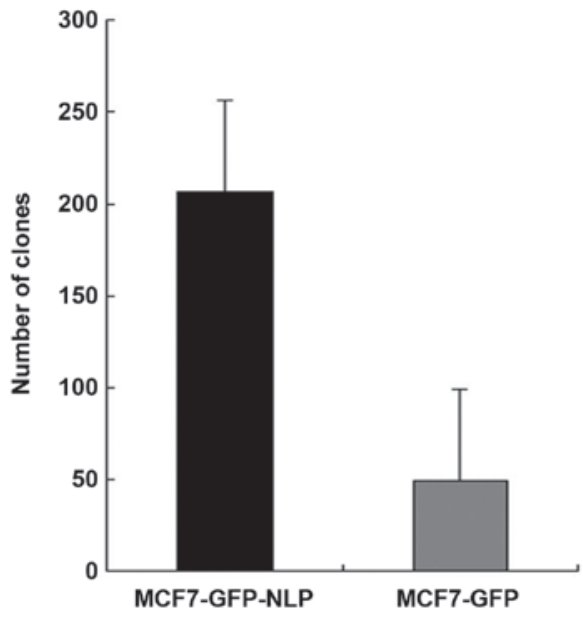

Figure 5. Colony numbers are increased in MCF7-GFP-NLP cells compared with MCF7-GFP cells $(\mathrm{P}<0.05)$. The data are expressed as the mean \pm standard error of the mean. GFP, green fluorescent protein; NLP, ninein-like protein.

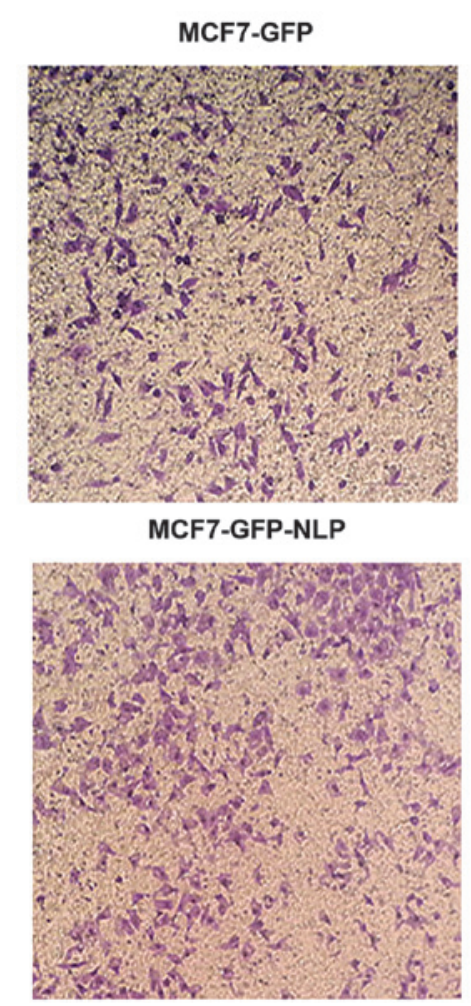

Figure 6. Levels of invasion, observed in a Transwell chamber, between the MCF7-GFP cells and MCF7-GFP-NLP cells. The migratory ability was increased in the MCF7-GFP-NLP cells, as compared with the MCF7-GFP cells. (Magnification, x100). GFP, green fluorescent protein; NLP, ninein-like protein.

adhering to the lower surface were fixed with methanol for 20 min, stained with 5\% Giemsa solution (Merck \& Co., Inc., Rahway, NJ, USA) for $20 \mathrm{~min}$ at room temperature, and quantified under a microscope (IX71; Olympus Corporation) in five randomly selected fields.

Statistical analysis. Statistical analyses were performed using SPSS 17.0 software (SPSS, Inc., Chicago, IL, USA) and all

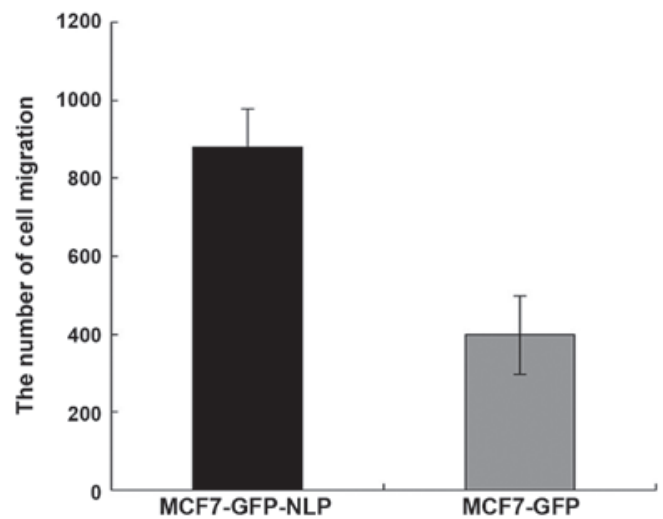

Figure 7. Increased expression of Nlp promotes the migration ability of MCF-7 cells $(\mathrm{P}<0.05)$. The data are expressed as the mean \pm standard error of the mean. GFP, green fluorescent protein; NLP, ninein-like protein.
1

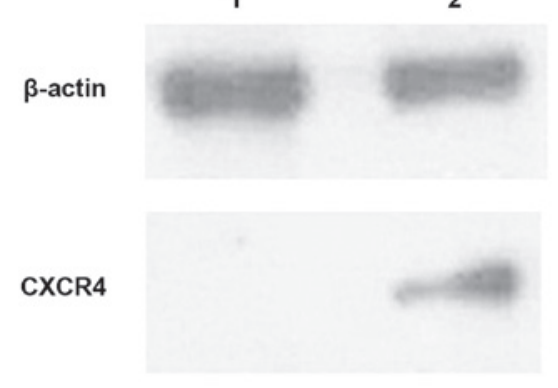

Figure 8. Detection of the expression of CXCR4 in (1) MCF7-GFP and (2) MCF7-GFP-Nlp cells, detected by western blot analysis. GFP, green fluorescent protein; NLP, ninein-like protein; CXCR, C-X-C chemokine receptor.

data are expressed as the mean \pm standard error of the mean. A least significant difference-t test was used for two sample comparisons from two groups. $\mathrm{P}<0.05$ was considered to indicate a statistically significant difference.

\section{Results}

Overexpression of Nlp is established in the MCF-7 breast cancer cell line. The pEGFP-C1-Nlp plasmid or the pEGFP-C1 plasmid were transfected into MCF-7 cells to establish MCF7-GFP-NLP and MCF7-GFP cells. The mRNA and protein expression levels of Nlp were detected by RT-qPCR and western blotting (Figs. 1 and 2).

Effect of high expression of Nlp on the growth of MCF-7 cells detected using an MTT assay. Under the same growth conditions, the growth rate was more rapid in the MCF7-GFP-NLP cells $(\mathrm{P}<0.05)$ compared with the MCF7-GFP cells, which indicated that Nlp promoted the growth of MCF-7 cells (Fig. 3).

Colony formation assay to detect cell proliferation ability. The results demonstrated that colony numbers in MCF-GFP cells and MCF7-GFP-NLP cells were $49 \pm 3.45$ and $206 \pm 14.35$, respectively, under identical conditions. The colony formation rate was markedly increased in the MCF7-GFP-NLP cells $(\mathrm{P}<0.05)$ compared with the MCF7-GFP cells, which indicated that Nlp promoted MCF7 cell proliferation (Figs. 4 and 5). 
Effect of high expression of Nlp on the migration ability in vitro. Under the same conditions, quantification of the cells migrated under the membrane in MCF7-GFP-Nlp cells and MCF7-GFP cells were $878 \pm 18.22$ and $398 \pm 8.02$, respectively. The migration ability was increased in the MCF7-GFP-Nlp cells compared with the MCF7-GFP cells and a significant difference was observed between two groups $(\mathrm{P}<0.05$; Figs. 6 and 7).

Expression of CXCR4 was detected by western blotting in the MCF7-GFP-Nlp cells and MCF7-GFP cells. The results of the western blotting revealed that the protein expression levels of CXCR4 were higher in the MCF7-GFP-Nlp cells compared with the MCF7-GFP cells (Fig. 8).

\section{Discussion}

Nlp is overexpressed in breast, lung and ovarian cancer, and head and neck squamous cell carcinoma. Notably, centrosomal Nlp causes spontaneous tumorigenesis in transgenic mice overexpressing Nlp (10-12). Previous studies have reported that centrosomal abnormalities occur in certain low-grade tumors and exhibit an demonstrate an increased trend in invasive tumors. In ovarian cancer tissues, the higher the pathological classification, the higher the number of centrosome abnormalities that are present. Notably, the presence of centrosome abnormalities are higher in malignant ovarian cancer (13-16). A previous study demonstrated that the overexpression of Nlp is observed in head and neck squamous cell carcinoma, which is associated with the clinicopathological characteristics (17). In addition, it has also been confirmed that the expression of Nlp significantly correlates with the tumor grade, and that the overexpression of Nlp is marginally associated with a decrease in overall survival rates (18). Furthermore, Nlp induces tumor development by interfering with the cell cycle, mitosis and cell apoptosis $(19,20)$. However, the effect of Nlp on breast tumor metastasis remains to be elucidated.

The MCF-7 breast cancer cell line retains several characteristics of differentiated mammary epithelium, including the ability to process estradiol via cytoplasmic estrogen receptors and the capability of forming domes. The present study established MCF7-GFP-Nlp and MCF7-GFP cells and observed, through growth curves that the MCF7-GFP-Nlp cells grew more rapidly compared with theMCF7-GFP cells. In addition, plate colony forming assays demonstrated that the MCF7-GFP-Nlp cells exhibited a increased colony formation capacity compared with the MCF7-GFP cells. These results indicated that Nlp promoted $\mathrm{MCF}-7$ cell proliferation. Transwell chambers are considered as a permeability support, and usually, a Transwell chamber is put into culture plates and medium is added to the top and bottom chambers, with the cells were seeded into the top chamber. Since the membrane is permeable, cells can migrate to the lower chamber $(21,22)$. The results of the present study revealed that the overexpression of Nlp promoted cell migration in the Transwell model in vitro.

Changes in tumor cell migration capacity is an important step affecting tumor invasion and metastasis. CXCR4 is encoded by 352 amino acids and is a seven-transmembrane G-protein chemokine receptor. In 1996, Feng et al identified that CXCR4 is a coreceptor for human immunodeficiency virus-1 entry, following which several studies have investigated CXCR4 (23). It has been demonstrated that CXCR4 is involved in the invasion and metastasis of several types of cancer, including breast carcinoma (24). Hiller and Chu $(25,26)$ demonstrated that CXCR4 is important in several types of cancer, including breast cancer, and revealed that CXCR4 was highly expressed in areas common for breast cancer metastasis, including the axillary lymph nodes. Hernandez et al confirmed that CXCL12-CXCR4 is important in the process of breast tumor cell growth, angiogenesis, invasion and metastasis $(27,28)$. A meta-analysis investigation based on thirteen eligible studies, consisting of 3,865 patients with breast cancer, demonstrated that the overexpression of CXCR4 was significantly associated with lymph node status and distant metastasis. In addition, the overexpression of CXCR4 indicated a poor overall and disease-free survival rates (29). The present study demonstrated that the expression of CXCR4 was higher in the MCF7-GFP-NLP cells compared with the MCF7-GFP control cells, which implied that Nlp improved the migration capacity of breast cancer cell lines through activated CXCL12 and CXCR4.

In conclusion, the results of the present study indicated that an increase in the expression of Nlp resulted in a malignant phenotype, which induced tumor cell proliferation and invasion. Furthermore, the results confirmed that Nlp exhibited certain biological characteristics, including promoting breast tumorigenesis and development, to provide a novel molecular index for breast cancer diagnosis. Therefore, Nlp may be an effective target of antitumor drugs for therapy against specific types of tumor.

\section{Acknowledgements}

This study was supported by the Science-Technology Development Funds of Shandong Province (no. 2011GSF11823).

\section{References}

1. Andre F, Slimane K, Bachelot T, et al: Breast cancer with synchronous metastases: trends in survival during a 14 -year period. J Clin Oncol 22: 3302-3308, 2004

2. Giordano SH, Buzdar AU, Smith TL, et al: Is breast cancer survival improving? Cancer 100: 44-52, 2004.

3. Li J and Zhan Q: The role of centrosomal Nlp in the control of mitotic progression and tumourigenesis. Br J Cancer 104: 1523-1528, 2011.

4. Bettencourt-Dias M and Glover DM: Centrosome biogenesis and function: Centrosomics brings new understanding. Nat Rev Mol Cell Biol 8: 451-463, 2007.

5. Doxsey SJ: Centrosomes as command centres for cellular control. Nat Cell Biol 3: E105-108, 2001.

6. Raff JW: Centrosomes: Central no more? Curr Biol 11: R159-R161, 2001.

7. Lingle WL, Lutz WH, Ingle JN, et al: Centrosome hypertrophy in human breast tumors: Implications for genomic stability and cell polarity. Proc Natl Acad Sci USA 95: 2950-2955, 1998.

8. Krämer A, Neben K and Ho AD: Centrosome aberrations in hematological malignancies. Cell Biol Int 29: 375-383, 2005.

9. Nigg EA: Origins and consequences of centrsome aberrations in human cancers. Int J Cancer 119: 2717-2723, 2006.

10. Shao S, Liu R, Wang Y, et al: Centrosomal Nlp is an oncogenic protein that is gene-amplified in human tumors and causes spontaneous tumorigenesis in transgenic mice. J Clin Invest 120: 498-507, 2010.

11. Qu D, Qu H, Fu M, et al: Increased expression of Nlp, a potential oncogene in ovarian cancer and its implication in carcinogenesis. Gynecol Oncol 110: 230-236, 2008. 
12. Yu L, Song Y, Zhang Q, et al: Ninein-like protein is overexpressed in head and neck squamous cell carcinoma and contributes to cancer growth and resistance to apoptosis. Oncol Rep 22: 789-798, 2009.

13. Pihan GA, Wallace J, Zhou Y, et al: Centrosome abnormalities and chromosome instability occur together in pre-invasive carcinomas. Cancer Res 63: 1398-1404, 2003.

14. Pihan GA, Purohit A, Wallace J, et al: Centrosome defects can account for cellular and genetic changes that characterize prostate cancer progression. Cancer Res 61: 2212-2219, 2001.

15. Duensing S: A tentative classification of centrosome abnormalities in cancer. Cell Biol Int 29: 352-359, 2005.

16. Siegel R, Naishadham D and Jemal A: Cancer statistics, 2012. CA Cancer J Clin 62: 10-29, 2012

17. Yu L, Song Y, Zhang Q, et al: Ninein-like protein is overexpressed in head and neck squamous cell carcinoma and contributes to cancer growth and resistance to apoptosis. Oncol Rep 22: 789-798, 2009.

18. Qu D, Qu H, Fu M, et al: Increased expression of Nlp, a potential oncogene in ovarian cancer and its implication in carcinogenesis. Gynecol Oncol 110: 230-236, 2008.

19. D'AssoroAB,LingleWLandSalisburyJL:Centrosomeamplification and the development of cancer. Oncogene 21: 6146-6153, 2002.

20. Duensing S: A tentative classification of centrosome abnormalities in cancer. Cell Biol Int 29: 352-359, 2005.

21. Wang Y, Yang H, Liu H, et al: Effect of staurosporine on the mobility and invasiveness of lung adenocarcinoma A549 cells: An in vitro study. BMC Cancer 9: 174, 2009.
22. Zhi YH, Song MM, Wang PL, et al: Suppression of matrix metalloproteinase-2 via RNA interference inhibits pancreatic carcinoma cell invasiveness and adhesion. World J Gastroenterol 15: 1072-1078, 2009.

23. Feng Y, Broder CC, Kennedy PE, et al: HIV-1 entry cofactor: Functional cDNA cloning of a seven-transmembrane, G protein-coupled receptor. Science 272: 872-877, 1996.

24. Juarez J, Bendall L and Bradstock K: Chemokines and their receptors as therapeutic targets: The role of the SDF-1/CXCR4 axis. Curr Pharm Des 10: 1245-1259, 2004.

25. Hiller D and Chu QD: CXCR4 and axillary lymph nodes: review of a potential biomarker for breast cancer metastasis. Int J Breast Cancer 420981, 2011.

26. Chu QD, Panu L, Holm NT, et al: High chemokine receptor CXCR4 level in triple negative breast cancer specimens predicts poor clinical outcome. J Surg Res 159: 689-695, 2010.

27. Hernandez L, Magalhaes MA, Coniglio SJ, et al: Opposing roles of CXCR4 and CXCR7 in breast cancer metastasis. Breast Cancer Res 13: R128, 2011.

28. Cojoc M, Peitzsch C, Trautmann F, et al: Emerging targets in cancer management: role of the CXCL12/CXCR4 axis. OncoTargets Ther 6: 1347-1361, 2013.

29. Zhang Z, Ni C, Chen W, et al: Expression of CXCR4 and breast cancer prognosis: a systematic review and meta-analysis. BMC Cancer 14: 49, 2014. 